\title{
Laser-Scanning Direction Effect in Femtosecond Laser-Assisted Etching
}

\author{
Shigeki Matsuo, Yoshifumi Umeda, Takuro Tomita, Shuichi Hashimoto \\ The University of Tokushima, 2-1 Minamijosanjimacho, Tokushima 770-8506, Japan. \\ E-mail: matsuo.shigeki@tokushima-u.ac.jp
}

\begin{abstract}
In this study we examined how the laser-scanning direction during the inscription of modified lines affects to the etching rate along the lines in the femtosecond laser-assisted etching. An isotropic material (silica) was used as the sample. Circular polarization was used for inscribing modified lines, in order to avoid a direction effect arising from polarization. Scanning direction dependence was observed in the etching rate along the modified lines. The relation between the occurrence of a scanning direction effect and the other inscribing parameters (pitch and pulse energy) was examined.
\end{abstract}

DOI:10.2961/jlmn.2013.01.0008

Keywords: femtosecond laser-assisted etching, scanning direction effect, quill effect, silica glass, $\mathrm{KOH}$, etching rate, pulse front tilt

\section{Introduction}

The development of femtosecond (fs) laser technology enabled new methods of micro-material processing such as the drilling of holes into metals with a minimized thermal effect [1] and three-dimensional (3D) photopolymerization [2]. In addition, by focusing fs pulses into transparent solid materials, we can modify material properties at the vicinity of the focus, without damaging the surface. This phenomenon is used for 3D optical recording [3] and inscribing optical waveguides [4].

Modification inside transparent solids is also used for removal processing combined with chemical etching. We call such a processing technique fs laser-assisted etching. First, the modified region along a predesigned pattern (e.g., lines and boxes) is inscribed using focused fs pulses by scanning the sample. The sample is then chemically etched. When the modified region is etched faster than the unmodified region, the etched region is preferentially dissolved, and a hollow structure inside the solid is fabricated. This is a unique technique because it allows the fabrication of hollow structures with 3D arbitrary designs and micrometer resolution $[5,6]$.

When fs laser pulses are used to modify a material, many processing parameters affect the modification, such as pulse duration, pulse energy, wavelength, repetition rate, and numerical aperture of the focusing optics. Polarization was also found to be an important parameter that affects the modification $[7,8]$. It was reported that scanning direction also affects the property of the modified region [9]. That is, a property (for example, appearance in optical microscopy) of the modified lines sometimes differs between lines inscribed in the opposite directions. Such an unexpected scanning direction effect is called the "quill effect" or "nonreciprocal writing," and has been reported by several authors [10-14].

Here we report the presence of a scanning direction effect on the femtosecond laser-assisted etching rate. Using circular polarization for the modification and an aqueous solution of potassium hydroxide $(\mathrm{KOH})$ as the etchant, a scanning direction effect was observed. We also examined the dependence of the occurrence of the scanning direction effect on the other irradiation parameters of pitch (the distance between neighboring irradiation points) and pulse energy.

\section{Experimental Methods}

\subsection{Laser irradiation}

Silica glass (Shin-Etsu Chemical Co., Ltd.) was used for the samples. This is an isotropic material, and thus we can avoid the direction effect due to the anisotropy of the samples.

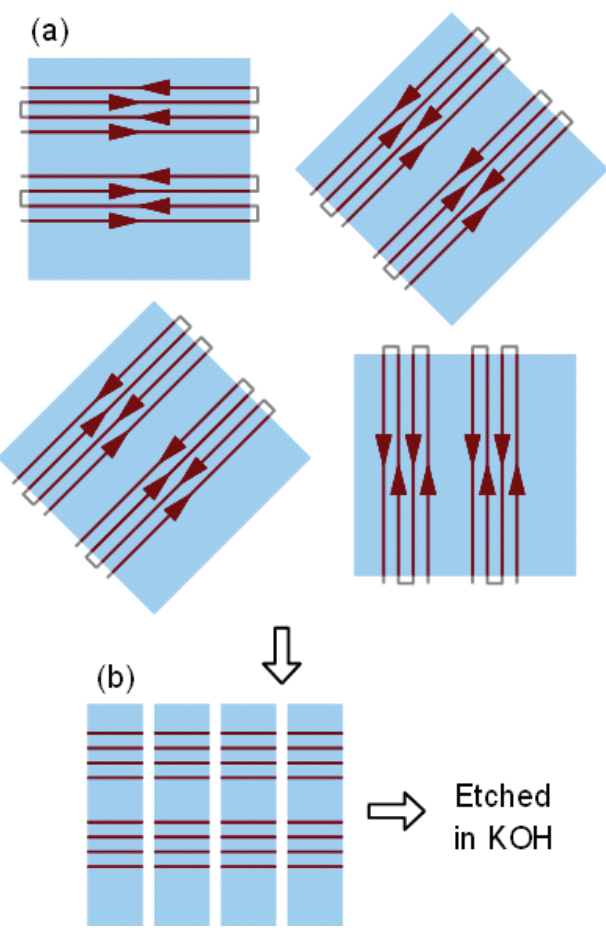

Fig. 1. Experimental procedure. (a) Modified lines were inscribed by irradiating focused fs pulses and translating the sample in alternating directions with different (i.e., pitch and pulse energy) values. (b) The sample was cut, and then it was etched in an aqueous solution of $\mathrm{KOH}$. 
First, modified lines were inscribed inside $10 \times 10-\mathrm{mm}$ silica substrates with the use of the fs laser. A Ti:Sapphire chirped-pulse-amplifier (Spitfire, Spectra-Physics), which emits 130-fs, 800-nm pulses with a maximum repetition rate of $1 \mathrm{kHz}$, was used as a light source. The emitted pulses were introduced to an optical microscope and focused into the sample by an objective lens $(40 \mathrm{x}, \mathrm{NA}=0.6)$. During irradiation, the sample was scanned to a specific direction with a motorized translation stage (KS701-20LHD, Suruga Seiki), so that modified straight lines were inscribed. Four lines were inscribed in a group in alternating directions (Fig. 1a) with a typical line interval of $50 \mu \mathrm{m}$. Several groups of lines are inscribed with different values (i.e., pitch and pulse energy). For different laser scanning directions, both the translation direction and the sample orientation were rotated (Fig. 1a). In the present experiments, the scanning was carried out in eight directions (every $45^{\circ}$ ) in the plane perpendicular to the optical axis. In order to avoid an effect of polarization, circularly polarized pulses were used for modification. The pitch was adjusted between 0.075 and $1.0 \mu \mathrm{m}$ by adjusting the sample translation speed and pulse repetition rate.

For the irradiation of the fs laser pulses into the silica sample, we used an optical microscope. The polarization of the pulses was adjusted before the microscope. The pulses were reflected by a dichroic mirror inside the microscope and then focused by an objective lens and irradiated into

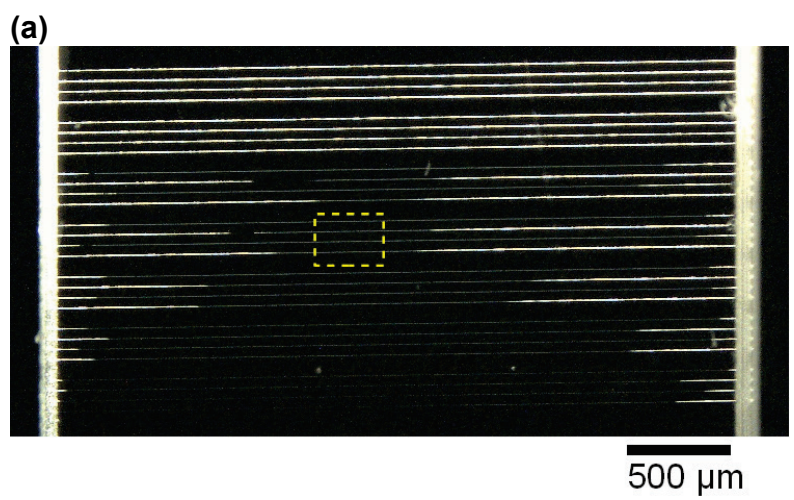

(b1)

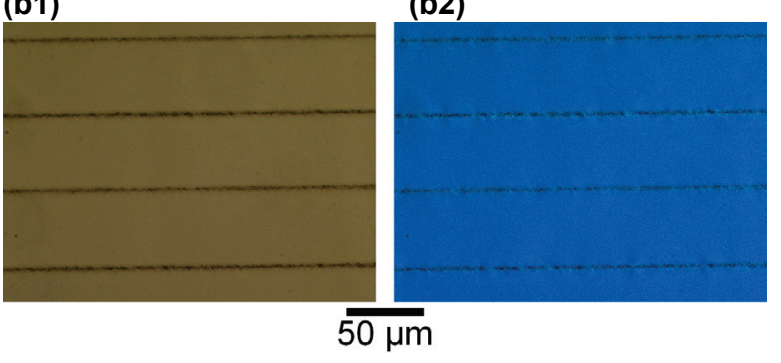

Fig. 2. (a) Optical micrograph of a sample after etching, taken with a wide-field microscope. Seven groups of lines were inscribed with different pulse energies. One group consisted of four lines; the four lines were inscribed by alternately changing the scanning directions at a constant pulse energy. The pulse energies of the seven groups were 1.2, 1.1, 0.8, 0.7, $0.6,0.5$, and $0.4 \mu \mathrm{J}$ (from the top to the bottom). The pitch was $0.1 \mu \mathrm{m}$. The sample was etched for $18 \mathrm{~h}$. (b) Close-up views of the rectangular region in (a) taken with a bright-field microscope (b1) and crosspolarized microscope (b2). the sample. In this case, the polarization at the focus might be affected by the dichroic mirror, because a dichroic mirror often has a birefringence; that is, a phase shift may occur between $s$ and $p$ polarizations. Due to this birefringence, even if the polarization was set as circular before the microscope, the polarization at the focus might not be circular. Hence we used a combination of a half-wave plate and a quarter-wave plate to obtain a circular polarization from linear polarization. The details of this procedure will be described elsewhere.

\subsection{Etching}

After the fs laser-inscribing of modified lines, the samples were cut perpendicular to the modified line (Fig. 1b) using a wire saw. Then, the sample was etched in a 1 $\mathrm{mol} /(\mathrm{dm})^{3}$ aqueous solution of $\mathrm{KOH}$ at $80^{\circ} \mathrm{C}$. The growth of the etched region along the modified lines was monitored in situ [15], and the etching rate was evaluated. For this evaluation, the growth of the etched region from the cut-edges - not from the original edges of the $10 \times 10 \mathrm{~mm}$ substrate - was measured in order to avoid the undesirable edge effect during the laser modification [16].

\section{Results and Discussion}

\subsection{Existence of laser-scanning direction effect}

The scanning direction effect was observed in our experiments. Figure 2a shows a typical optical micrograph of a sample after etching for $18 \mathrm{~h}$. Seven groups of lines were inscribed with different pulse energies. In the figure, the etched region along the inscribed lines appears brighter. The length of the etched region varied alternately in the third to sixth groups (pulse energy range, $0.5-0.8 \mu \mathrm{J}$ ); shorter in the first and third lines and longer in the second and fourth lines of each group (Fig. 2a). This result shows the occurrence of the scanning direction effect in the etching rate. It should be pointed out that the etching rate from both edges showed the same tendency. This indicates that the difference between lines is not directional (e.g., a line inscribed in the $+x$ direction is etched faster in the $+x$ direction but etched slower in the $-x$ direction), but rather it is quantitative (e.g., a line inscribed in the $+x$ direction is etched faster in both the $+x$ and $-x$ directions, and a line inscribed in the $-x$ direction is etched slower in both directions).

As mentioned previously, we used circular polarization for inscribing modified lines in the present experiments. To the best of our knowledge, this is the first observation of a scanning direction effect using circular polarization.

Figure $2 \mathrm{~b}$ shows close-up views of modified but not yet etched regions; (b1) is a bright field micrograph and (b2) is a cross-polarized micrograph. With these we can compare the appearance between the opposite scanning directions (top and third lines = slower-etched; second and fourth lines $=$ faster-etched). In both b1 and b2, the difference between the appearance of the slower-etched and fasteretched lines is not significant. This result indicates that the etching is a sensitive method to visualize a scanning direction effect. 


\subsection{Dependence of eight-direction etching rates on ir- radiation parameters}

The etching rates along the eight scanning directions were evaluated, and they were compared among the different (pitch and pulse energy) values. The reproducibility of the results is not yet adequate for quantitative conclusions; here we qualitatively summarize the results.

1. Figure 3 shows a typical radar chart of etching rates. There was a "fast" direction (right in Fig. 3) and a "slow" direction (left in Fig. 3) that was opposite to the "fast" direction. Between the "fast" and "slow" directions, the etching rate varied monotonically.

2. An inversion of "fast" and "slow" directions was observed with a change in pulse energy, whereas the "fast-slow" axis was kept constant.

3. The occurrence of the scanning direction effect depended on the pitch, $p$. In short pitch range (ca. $p \leq 0.15 \mu \mathrm{m}$ ), the scanning direction effect was significant, and in the moderate pitch range (ca. $0.15 \mu \mathrm{m}<p \leq$ ca. $0.6 \mu \mathrm{m}$ ), the effect was less significant. In the longer pitch range, the etching rate decreased and finally fell to zero. This tendency (in which a direction dependence is significant in the short pitch range) is consistent with previous reports $[9,13]$, whereas the range of pitch differed. In addition, the etching rate was higher in the moderate pitch range than the short pitch range. Thus the moderate pitch range is adequate for practical use.

\subsection{Origin of scanning direction dependence}

Because we used an isotropic material for the samples, the origin of the scanning direction dependence is probably attributable to the anisotropy of i) the irradiated laser pulse and/or ii) the translation stage, but the latter is less probable. Kazansky et al. proposed that pulse-front-tilt - which often and unintentionally arises in fs laser pulses - is the origin of scanning direction dependence [9]. In our experiments, the orientation of the "fast-slow" axis was constant, and was in agreement with the orientation in which pulsefront-tilt is expected to occur with our laser setup. To further examine the origin of the scanning direction dependence, it is desirable to measure pulse-front-tilt as well as the other possible anisotropy of fs laser pulses such as spa-

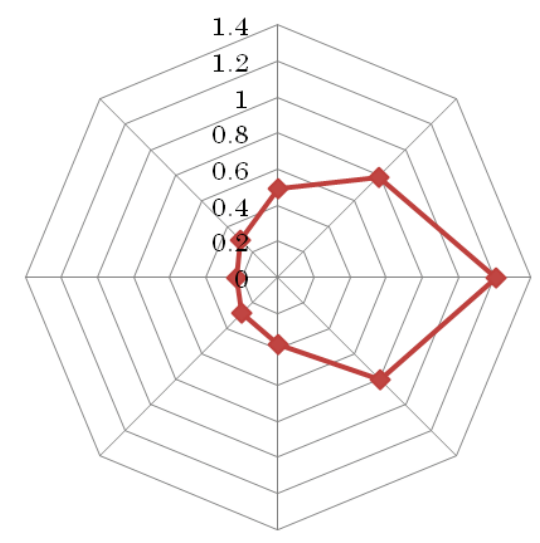

Fig. 3. A radar chart showing scanning angle dependence of the etching rate (the unit was $\mu \mathrm{m} / \mathrm{min}$ ). The pitch was $0.1 \mu \mathrm{m}$ and the pulse energy was $0.7 \mu \mathrm{J}$. tial intensity distribution.

In the present experiments, we used circular polarization for inscribing modified lines. For circular polarization, formation-disordered and interconnected nano-void structure has been reported [17], in contrast to nano-gratings in the case of linear polarization. We speculate that the random nano-void structure differs slightly among the laserscanning directions, and the etching rate is sensitive to the slight difference in nano-structure.

\section{Conclusion}

We have studied the laser-scanning direction effect in the fs laser-assisted etching of an isotropic material of silica with circular polarization. Scanning direction dependence was clearly observed in the etching rate. The dependence was significant in the short pitch range. In the moderate pitch region, the etching rate was higher and less dependent on the scanning direction, and thus the moderate pitch range is adequate for practical use.

\section{Acknowledgments}

We thank Prof. Y. Bellouard (Eindhoven University of Technology, the Netherlands) for the fruitful discussions. This work was partly supported by KAKENHI (20360115).

\section{References}

[1] B.N. Chichkov, C. Momma, S. Nolte, F.V. Alvensleben, and A. Tünnermann, Appl. Phys. A, 63, (1996) 109

[2] S. Maruo, O. Nakamura, and S. Kawata, Opt. Lett., 22, (1997) 132.

[3] E.N. Glezer, M. Milosavljevic, L. Huang, R. J. Finlay, T.-H. Her, J. P. Callan, E. Mazur, Opt. Lett., 21, (1996) 2023.

[4] K. Miura, J. Qiu, H. Inouye, T. Mitsuyu, and K. Hirao, Appl. Phys. Lett., 71, (1997) 3329.

[5] Y. Kondo, J. Qiu, T. Mitsuyu, K. Hirao, T. Y. Oko, and T. Yoko, Jpn. J. Appl. Phys., 38, (1999) L1146.

[6] A. Marcinkevičius, S. Juodkazis, M. Watanabe, M. Miwa, S. Matsuo, H. Misawa, J. Nishii, Opt. Lett., 26 (2001) 277.

[7] Y. Shimotsuma, P. G. Kazansky, J. Qiu, and K. Hirao, Phys. Rev. Lett., 91, (2003) 247405.

[8] C. Hnatovsky, R.S. Taylor, E. Simova, V.R. Bhardwaj, D. M. Rayner, and P. B. Corkum, Opt. Lett., 30, (2005) 1867

[9] P.G. Kazansky, W. Yang, E. Bricchi, J. Bovatsek, A. Arai, Y. Shimotsuma, K. Miura, K. Hirao, Appl. Phys. Lett., 90, (2007) 151120.

[10] W. Yang, P.G. Kazansky, Y. Shimotsuma, M. Sakakura, K. Miura, and K. Hirao, Appl. Phys. Lett., 93, (2008) 171109.

[11] W. Yang, P.G. Kazansky, and Y. P. Svirko, Nature Photonics, 2, (2008) 99.

[12] B. Poumellec, M. Lancry, J.-C. Poulin, and S. AniJoseph, Opt. Express, 16, (2008) 18354.

[13]D. N. Vitek, E. Block, Y. Bellouard, D.E . Adams, S. Backus, D. Kleinfeld, C. G. Durfee, and J. A. Squier, Opt. Express, 18, (2010) 24673.

[14] Y. Bellouard and M.-O. Hongler, Opt. Express, 19, (2011) 6807-6821. 
[15] S. Matsuo, H. Sumi, S. Kiyama, T. Tomita and S. Hashimoto, Appl. Surf. Sci., 255, (2009) 9758-9860.

[16] C. Hnatovsky, R.S. Taylor, E. Simova, P.P. Rajeev, D. M. Rayner, V.R. Bhardwaj, and P.B.Corkum, Appl. Phys. A, 84, (2006) 47.
[17]C. Hnatovsky, E. Simova, P.P. Rajeev, D.M. Rayner, P.B. Corkum, and R.S. Taylor, Opt. Lett., 32, (2007) 1459 .

(Received: June 30, 2012, Accepted: November 19, 2012) 